\title{
Isolated Bradycardia During Aneurysmal Clipping: Rebleed or Trigeminocardiac Reflex?
}

\author{
Nidhi Singh ${ }^{1}$ Kiran Jangra ${ }^{1}$ Sabina Regmi ${ }^{1}$ Apinderpreet Singh ${ }^{2}$ \\ ${ }^{1}$ Department of Anaesthesia and Intensive Care, Post Graduate \\ Institute of Medical Education and Research, Chandigarh, India \\ ${ }^{2}$ Department of Neurosurgery, Post Graduate Institute of Medical \\ Education and Research, Chandigarh, India

\begin{abstract}
Address for correspondence Kiran Jangra, DM, Department of Anaesthesia and Intensive Care, 4th Floor, Nehru Hospital, Post Graduate Institute of Medical Education and Research, Chandigarh 160012, India (e-mail: drkiransharma0117@gmail.com).
\end{abstract}

\begin{abstract}
Keywords

- bradycardia

- Cushing's traid

- intracranial aneurysm

- rebleeding

- trigeminocardiac reflex

The most common cause of nontraumatic subarachnoid hemorrhage is the rupture of intracranial aneurysm. After initial bleed, the risk of rebleeding is highest in the early postictal period and this rebleed is strongly associated with poor neurological outcome. The major goal of anesthesia in these surgeries is to prevent the rebleed. If rebleeding occurs prior to the craniotomy, it results in the acute rise of intracranial pressure and usually presents as bradycardia and hypertension (Cushing's reflex). Here we reported a case where rebleeding presented unusually as isolated bradycardia without associated hypertension and was mistaken as trigeminocardiac reflex. The surgeon was informed about the event and they planned to proceed. After craniotomy, despite all the efforts the brain was persistently tight and surgery could not be completed. Postoperative scan showed rebleeding and the patient died after a few days in ICU.

We highlighted in this case report the fact that isolated transient bradycardia may also be the presentation of rebleed with closed cranial vault. It is not always necessary to see all the features of Cushing's traid in every patient. If bradycardia occurs before the craniotomy, the surgeon should be notified, the severity of bleed should be assessed, and further management should be planned according to the severity of bleed.
\end{abstract}

\section{Introduction}

Aneurysmal bleed is the most common nontraumatic cause of subarachnoid hemorrhage. After initial bleed, the risk of rebleeding is highest in first 72 hours with an incidence of 8 to $23 \% .^{1}$ Rebleeding is a devastating complication of aneurysmal subarachnoid hemorrhage and is strongly associated with poor neurological outcome. Perioperatively, rebleed may occur at any point of time and usually presents as bradycardia and hypertension due to the acute rise in intracranial pressure (ICP) before cranium is opened. Here we report a case where we could not recognize the rebleed intraoperatively as it presented as isolated bradycardia and was not associated with hypertension.

\section{Case Report}

A 40-year-old man with ruptured right internal carotid artery (ICA) bifurcation saccular aneurysm of $6.9 \times 3.3 \mathrm{~mm}$

Published online

June 1, 2020
DOI https://doi.org/

10.1055/s-0040-1710298

ISSN 2348-0548. in size, with a neck of $4.7 \mathrm{~mm}$, was posted for aneurysmal clipping. On presentation, patient had Hunt and Hess (H\&H) grade 2, World Federation of Neurosurgical Sciences grade 1, and modified Fisher grade 1. On examination, he was conscious, oriented with Glasgow coma scale score of 15 , and had stable vitals. Bilateral pupils were reacting to light and he was moving all four limbs equally. All the preoperative investigations were within normal limits.

The patient was taken in operation theater and standard American Society of Anesthesiologist monitors including electrocardiography, pulse oximetry, and noninvasive blood pressure were attached. Baseline vitals were stable with heart rate (HR) $85 \mathrm{bpm}$, blood pressure (BP) 120/70 mm Hg, oxygen saturation $99 \%$ on room air, and respiratory rate 16 per minute. Anesthesia was induced using injection fentanyl $100 \mu \mathrm{g}$ and propofol titrated to loss of consciousness, followed by infusion targeted to achieve entropy of 40 to 60 . To facilitate endotracheal intubation, injection vecuronium 
$6 \mathrm{mg}$ was given. To prevent intubation response, additional bolus of propofol $40 \mathrm{mg}$ was given. After confirming correct tube placement, patient was kept on volume-controlled ventilation with ventilator settings targeted to keep end-tidal carbon dioxide $\left(\mathrm{EtCO}_{2}\right)$ around $35 \mathrm{~mm} \mathrm{Hg}$. Post intubation, arterial cannula and central line were inserted. The patient was maintained on propofol with oxygen:nitrous oxide 1:1 titrated to the entropy values of 40 to 60 . The antibiotic and mannitol $60 \mathrm{~g}$ were given as part of our institutional practice.

The head was fixed in extended position using four-pin head holder after lignocaine infiltration at pin site and additional propofol bolus (50 mg), to prevent the hemodynamic response. During head positioning, there was a transient episode of bradycardia from $85 \mathrm{bpm}$ to $42 \mathrm{bpm}$, BP 134/90 mm $\mathrm{Hg}$ which resolved within seconds without any intervention. The patient had another episode of severe bradycardia up to 38 bpm with BP of 127/74 mm Hg (-Fig. 1) during drilling the burr hole. As blood pressure was stable, no intervention was done and the surgeons were informed.

After the bone flap elevation, the surgeon observed that the duramater was tense. Various measures were taken to relax the brain including head end elevation, switching off nitrous oxide, hyperventilation to achieve $\mathrm{EtCO}_{2}$ of $30 \mathrm{~mm}$ $\mathrm{Hg}$, checking airway pressures, repeated the dose of mannitol, and supplemented anesthesia with boluses of muscle relaxant and propofol. Despite all these measures, the condition of brain did not improve. Thereafter the surgeon decided to release the cerebrospinal fluid (CSF), and $20 \mathrm{~mL}$ of CSF was drained by putting brain cannula in the lateral ventricle. Even after the drainage of CSF, the brain did not shrink. Then, temporal lobectomy was attempted, and that also failed to relax the brain. During this entire event the blood pressure of patient remained within $10 \%$ of baseline. Ultimately, a joint decision was made to abandon the procedure. A decompressive craniotomy was done, bone flap was placed in the abdomen, and the patient was shifted to intensive care unit. Postoperative computed tomography revealed increase in the amount of bleed in the fissures and a diagnosis of intraoperative rebleed of aneurysm was made (-Fig. 2). The patient had a ventilatory stay of 10 days, and later died due to severe vasospasm.

\section{Discussion}

Out of various causes of intraoperative bradycardia reported in neurosurgical literature, trigeminocardiac reflex (TCR), direct brainstem handling, and Cushing's reflex secondary to sudden rise in ICP are frequently obeseved. ${ }^{2}$ The Cushing's reflex, also referred to as the vasopressor response, is characterized by occurrence of the triad of hypertension, bradycardia, and irregular breathing. ${ }^{3}$ TCR usually manifests as a sudden onset of negative hemodynamic influences on HR and BP, and has been associated with severe bradycardia, cardiac arrhythmias, and asystole. The classic definition includes a decrease in HR and mean arterial blood pressure, and the stimulation of sensory component of trigeminal nerve may lead to tachycardia and hypertension. ${ }^{4}$ There are various causes of intraoperative IAR enumerated in - Table 1. All these causes were ruled out in our case.

Intraoperative recognition of rerupture of aneurysm through clinical signs can be perplexing. During steady state anesthesia, intraoperative aneurysm rupture (IAR) prior to craniotomy causes acute rise in ICP that manifests as sudden rise in BP with a precipitous fall in HR. In the present case, bradycardia was transient and was an isolated finding; hence, we could not recognize this complication at first place. All parameters of the Cushing's triad are sometimes not present. ${ }^{5}$
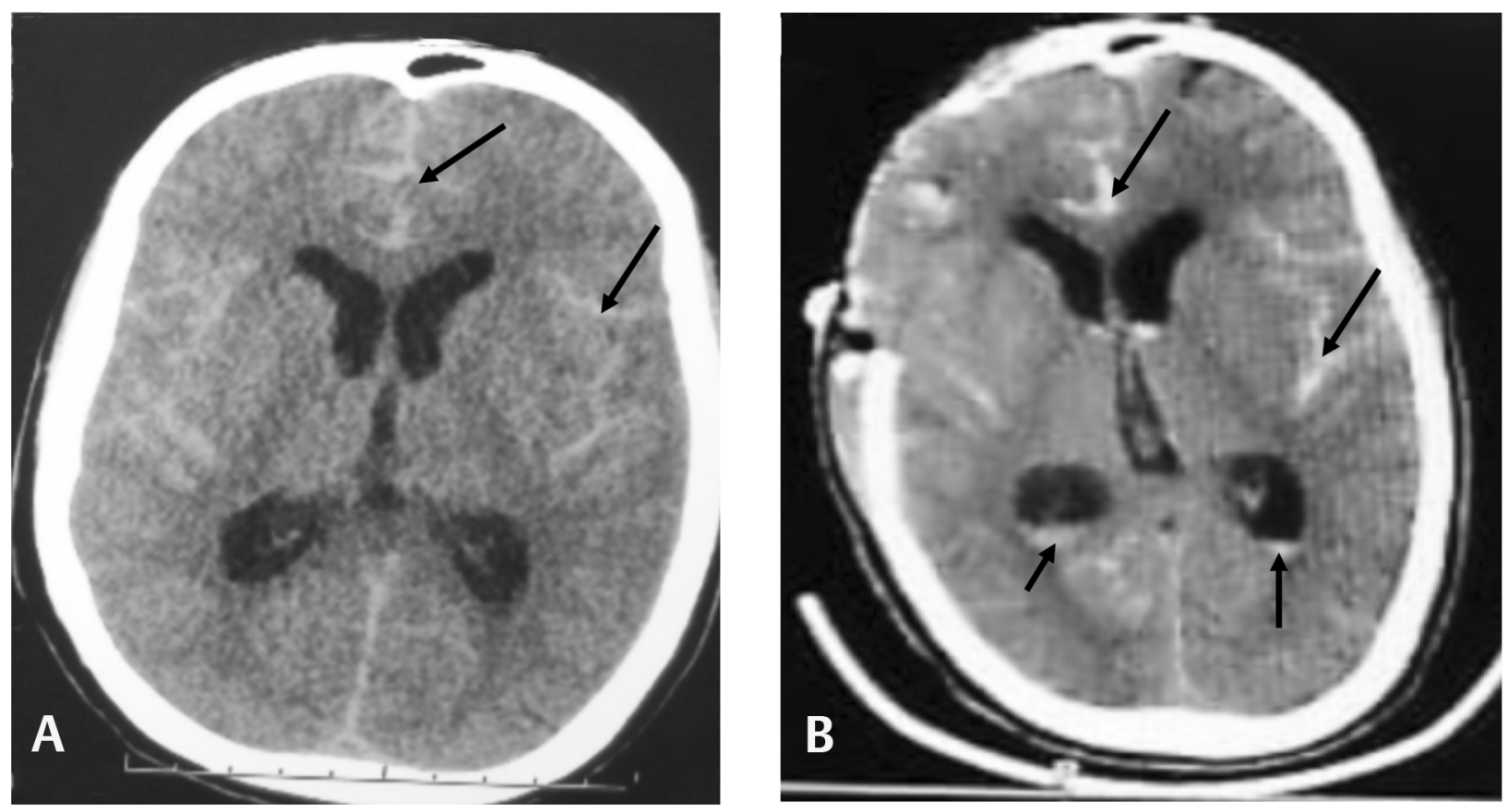

Fig. 1 (A) Preoperative noncontrast computed tomography scan showing subarachnoid hemorrhage indicated by black arrows. (B) Postperative noncontrast computed tomography scan showing increased severity of subarachnoid hemorrhage with intraventricular hemorrhage. 


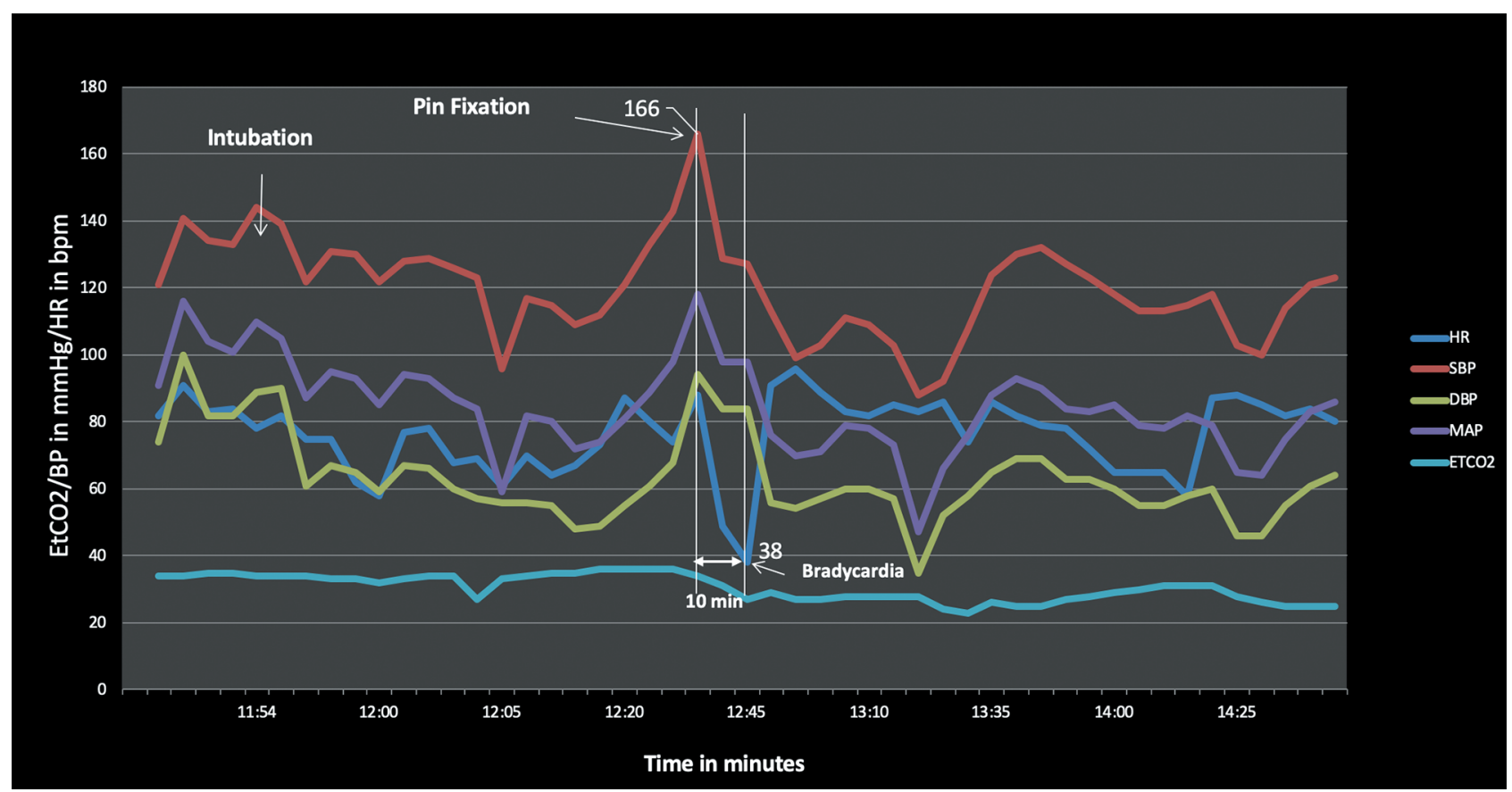

Fig. 2 Intraoperative vital trends. The events are marked by white arrows. DBP, diastolic blood pressure; EtCO2, endtidal carbon dioxide; $H R$, heart rate; MAP, mean arterial pressure; SBP, systolic blood pressure.

Table 1 Causes and risk factors of intraoperative aneurysm rupture

\begin{tabular}{|ll|}
\hline 1. & Poorly controlled blood pressure \\
\hline 2. & $\begin{array}{l}\text { Sympathetic responses (intubation, head pin fixation, } \\
\text { craniotomy, extubation) }\end{array}$ \\
\hline 3. & Coughing and bucking \\
\hline 4. & Sudden decrease in intracranial pressure \\
\hline 5. & Valsalva manoeuvre \\
\hline 6. & Application of positive end expiratory pressure \\
\hline 7. & Patients with history of chronic obstructive pulmonary \\
disease, coronary artery disease, and hyperlipidemia
\end{tabular}

Acute intracranial bleed causes sudden rise in ICP that may act as a tamponade to decrease the evolving bleed. Consequently, rapidly decreasing ICP in presence of IAR is open to discussion. ${ }^{6}$ In this case also, draining CSF by surgeons to decrease ICP may have further aggravated the bleed. Absence of hypertension in this case may be because of the additional propofol boluses that were repeated frequently to avoid pin response and then to relax the brain. Also, the stunning of myocardium could not be ruled out in the absence of echocardiography findings.

There are various stages prior to craniotomy when aneurysm can rebleed such as intubation, pin fixation, making burr hole, and drilling. During these stages, depth of anesthesia and relaxation should be adequate and additional boluses of various drugs, such as propofol, fentanyl, and lignocaine (intravenous or local site infiltration), may be supplemented to control the sympathetic response, if needed.

\section{Conclusion}

With this case report, we would like to highlight that even transient bradycardia before opening duramater along with a tense brain may be an indicator of aneurysmal rebleed. An urgent CT scan may be acquired before proceeding further.

\section{Conflict of Interest}

None declared.

\section{References}

1 Larsen CC, Astrup J. Rebleeding after aneurysmal subarachnoid hemorrhage: a literature review. World Neurosurg 2013;79(2):307-312

2 Faried A, Sendjaja AN, Melia R, Arifin MZ. Bradycardia without hypertension and bradypnea in acute traumatic subdural hematoma is a sensitive predictor of the Cushing triad: 3 case reports. Interdiscip Neurosurg 2016;6:84-86

3 Yumoto T, Naito H, Yorifuji T, et al. Cushing's sign and severe traumatic brain injury in children after blunt trauma: a nationwide retrospective cohort study in Japan. BMJ Open 2018;8(3):e020781

4 Leon-Ariza DS, Leon-Ariza JS, Nangiana J, Vargas Grau G, LeonSarmiento FE, Quiñones-Hinojosa A. Evidences in neurological surgery and a cutting edge classification of the trigeminocardiac reflex: a systematic review. World Neurosurg 2018;117:4-10

5 Radhakrishnan N, Chouhan RS, Kapoor I, Mahajan C. Intraoperative predissection aneurysm rupture: no less than a catastrophe! J Neuroanaesth Crit Care 2019. Doi: 10.1055/s-0039-1700588

6 Chowdhury T, Petropolis A, Wilkinson M, Schaller B, Sandu N, Cappellani RB. Controversies in the anesthetic management of intraoperative rupture of intracranial aneurysm. Anesthesiol Res Pract 2014;2014:595837 